\title{
A new ground-to-train communication system using free-space optics technology
}

\author{
H. Kotake, T. Matsuzawa, A. Shimura, S. Haruyama \\ \& M. Nakagawa \\ Department of Information and Computer Science, Keio University, \\ Japan
}

\begin{abstract}
We propose a new ground-to-train communication system using free-space optics between a train and the ground. In the proposed system, a cylindrical concave lens spreads the incoming beam from transmitter (Laser Diode, LD) horizontally to form a wide fan-shaped beam. The fan-shaped beam is projected to a train and the width of the projected beam is equal to the length of a typical bullet-train car. This transmitter with cylindrical concave lens and a receiver (Avalanche Photo Diode, APD) are installed on a train and the ground, and the horizontally spread beam is received continuously by the corresponding receiver. The train can keep a communication link continuously to the ground thanks to this spread beam. We analyzed the performance of the proposed system by experiments. The experimental results show that a sufficient received Signal-toNoise Ratio (SNR) can be obtained when a train is not moving; therefore, the signal can be received continuously even when the train is moving. Furthermore, in order to keep a continuous communication link even when the train vibrates or shifts vertically, the vertical spread angle of a laser beam is adjusted between 0.1 degrees and 0.5 degrees. These experimental results lead to the conclusion that this system is expected to be a Giga-bit class high speed communication technology between the train and the ground.

Keywords: train communication, optical wireless communication, visible light communication, horizontally spread beam.
\end{abstract}

\section{Introduction}

In recent years, the environment of the ubiquitous society is being developed by rapid expansion of high-speed communication infrastructure such as Asymmetric 
Digital Subscriber Line (ADSL) and Fiber To The Home (FTTH). We can use many services in hotspot and internet cafe. There is a high demand for the infrastructure to provide enough service to customers in a train. Leaky CoaXial cable (LCX) and millimeter-wave have been used for wireless communication between a train and the ground. However, the data rate of LCX is only 2.56 Mbps [1]. Millimeter-wave can increase the data rate up to $1 \mathrm{Gbps}$ when a train is not moving, but the data rate is decreased to $6.3 \mathrm{Mbps}$ when the train is moving [2]. Therefore, these systems cannot provide high-speed data transmission in the moving train.

The equipments of optical wireless communication such as LD and LED are developed rapidly, and it is possible to achieve high-speed communication with these equipments. Since frequency of lightwave is very high, optical wireless communication is suitable for high-speed communication [3, 4].

We propose a new train communication system using free-space optics technology between a train and the ground. In the proposed system, a cylindrical concave lens spreads horizontally the incoming beam from LD horizontally to form a wide fan-shaped beam. The fan-shaped beam is projected to a train and the width of the projected beam is $25 \mathrm{~m}$ which is equal to the length of a typical bullet-train car. Owing to this spread beam, the train can continuously keep a communication link to the ground.

In this paper, we analyze the performance of this system experimentally. We investigate the received SNR in stationary condition and received signal amplitude at moving environment. Moreover, we examine the received power with the expansion of vertical beam angle in order to have a continuous communication link. Finally, we conducted the outdoor experiment using the test train.

The rest of the paper is organized as follows. In section 2, we describe LD with variable Numerical Aperture (NA) lens and cylindrical concave lens. In section 3, we describe the proposed system model. In section 4 , we evaluate the system performance experimentally. Finally, the conclusions are given in section 5 .

\section{Design of transmitter}

\subsection{LD fixed with variable NA lens}

LD is a device that emits the light by induced emission and laser oscillation and outputs the coherent light. The light of LD has isolated wavelength and high directivity, and the transmission power of LD is higher than that of LED $[5,6]$. Even though the light of LD has high directivity, the width of the beam projected to a train is diffused and the sufficient received optical power cannot be obtained.

To obtain sufficient power of LD at the train, a variable NA lens which can change focal distance is attached in front of the LD. Since the incident beam angle from LD can be adjusted, the transmitted power can be controlled locally. Figure 1 shows the photograph and the concept of LD fixed with variable NA lens. The vertical beam angle of this LD is set to between 0.1 and 5.72 degrees, and the horizontally spread beam angle is fixed to 4.62 degrees. 


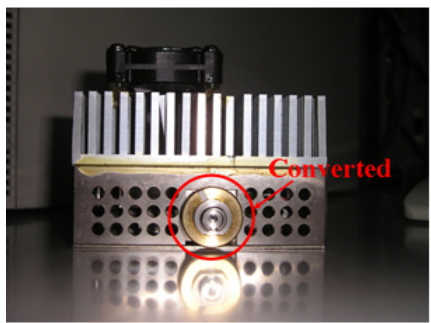

(Photograph)

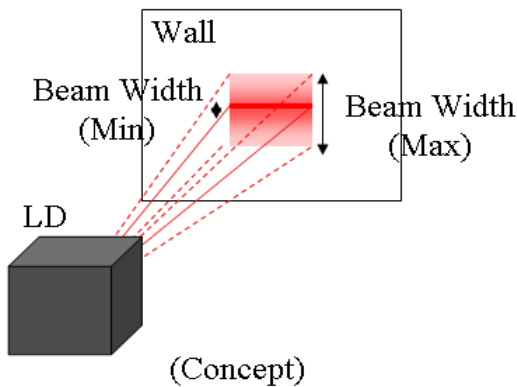

Figure 1: Photograph and Concept of LD fixed with variable lens.

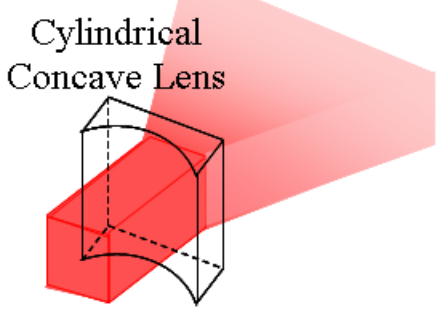

Figure 2: Effect of cylindrical concave lens.

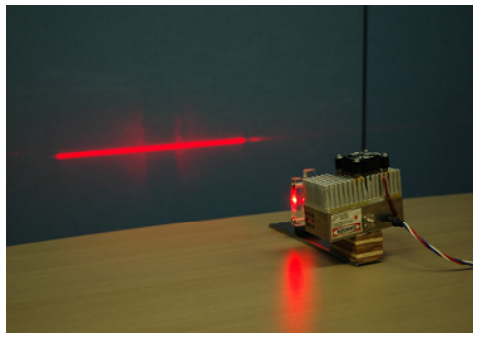

Figure 3: Photograph of laser beam which is spread by cylindrical concave lens.

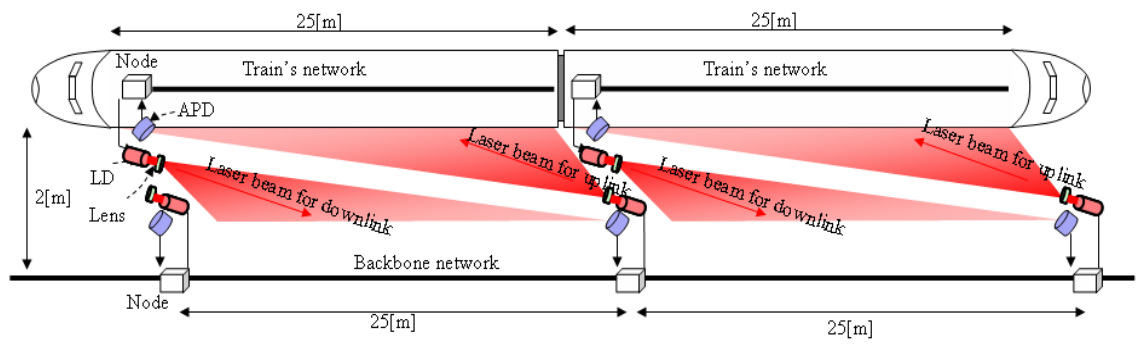

Figure 4: $\quad$ Proposed system model. 


\subsection{Cylindrical concave lens}

Various types of lens are categorized by configuration. There are many types of lens such as spherical lens, paraboloidal lens, and cylindrical lens. Among various types, cylindrical lens have a shape of cylinder, and can bring about an effect of lens in one direction. Since horizontally spread beam is projected to a train in the proposed system, we select cylindrical concave lens which spreads horizontally the incoming beam from LD to form a wide fan-shaped beam. Figure 2 shows the effect of cylindrical concave lens, and Figure 3 shows the photograph of a laser beam which is spread by the cylindrical concave lens. In this paper, we select three beam angles spread horizontally by cylindrical concave lens such as 20,30, and 40 degrees.

\section{Description of proposed system}

\subsection{System model}

The proposed system model is illustrated in Figure 4. The width of the projected beam is $25 \mathrm{~m}$ which is equal to the length of a typical bullet-train car. Since the horizontal distance between a bullet-train and LCX is $1.9 \mathrm{~m}$, if it is possible to communicate at this distance, LD and APD can be installed instead of LCX. Because of this, the horizontal distance between train and ground is $2 \mathrm{~m}$ (the height of LD and APD is the same). The down-link communication from the ground to the train and the up-link communication from the train to the ground use the same transmitter (LD) and receiver (APD) so that the speed of down-link and up-link are designed to be the same.

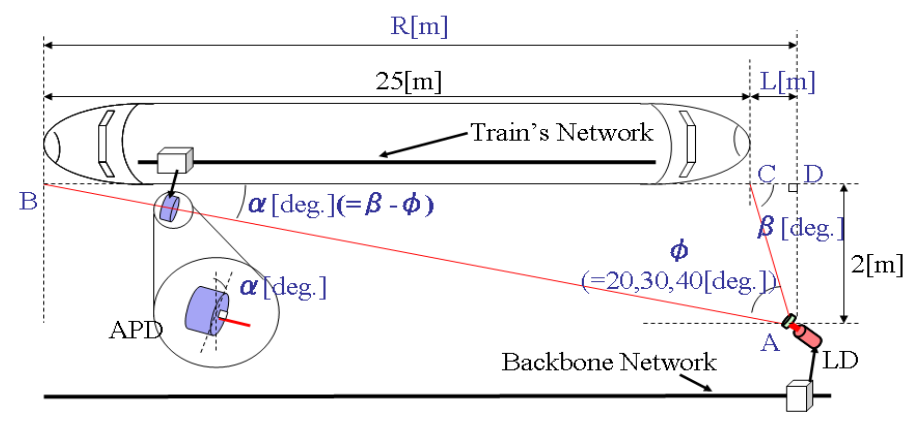

Figure 5: $\quad$ Proposed layout model.

\subsection{Layout model}

The proposed layout model is shown in Figure 5. Figure 5 shows only downlink, but up-link is also designed in a similar manner. We calculate various parameters to set up the layout. The calculated values of the various parameters with the width of the projected beam of $25 \mathrm{~m}$ are listed in Table 1 , where $\varphi$ is 
horizontally spread beam angle and is set to 20,30, 40 degrees, $\alpha$ is the angle which received at the longest point $B$ and $\beta$ is the angle which received at the shortest point $\mathrm{C}$. In other words, $\alpha$ is the angle at which APD is tipped. L is the distance between point $\mathrm{C}$ and $\mathrm{D}$, and $\mathrm{R}$ is the distance between point $\mathrm{B}$ and $\mathrm{D}$.

Table 1: $\quad$ Parameters of layout model.

\begin{tabular}{|c|c|c|c|}
\hline$\varphi$ (degrees) & 40 & 30 & 20 \\
\hline$\alpha$ (degrees) & 4.3 & 4.1 & 3.9 \\
\hline$\beta$ (degrees) & 44.3 & 34.1 & 23.9 \\
\hline $\mathrm{L}(\mathrm{m})$ & 2.05 & 2.95 & 4.51 \\
\hline $\mathrm{R}(\mathrm{m})$ & 27.05 & 27.95 & 29.51 \\
\hline $\mathrm{AB}(\mathrm{m})$ & 27.12 & 28.02 & 29.58 \\
\hline $\mathrm{AC}(\mathrm{m})$ & 2.86 & 3.56 & 4.93 \\
\hline
\end{tabular}

Table 2: $\quad$ Experimental parameter.

\begin{tabular}{|c|c|}
\hline Bit rate & $100(\mathrm{Mbps})$ \\
\hline Transmission power of LD & $100(\mathrm{~mW})$ \\
\hline Wavelength of LD & $670(\mathrm{~nm})$ \\
\hline Frequency of LD & $400(\mathrm{MHz})$ \\
\hline Horizontally spread beam angle (with lens) & $20 / 30 / 40($ degrees $)$ \\
\hline Vertical beam angle & $0.1-5.72($ degrees $)$ \\
\hline Receiver & APD \\
\hline Diameter of receiver & $0.5(\mathrm{~mm})$ \\
\hline Material of cylindrical concave lens & Synthetic fused silica \\
\hline Condition of room & Dark room \\
\hline Received signal amplitude of ambient noise & $4(\mathrm{mV})$ \\
\hline
\end{tabular}

\section{Experimental results}

In this section, we evaluated the system performance experimentally. In particular, we show the received SNR in a stationary condition, received signal amplitude in a moving condition, the received power with the expansion of vertical beam angle. Table 2 shows experimental setup.

\subsection{Received SNR in a stationary condition}

Measurement environment and result of the received SNR in stationary condition are shown in Figure 6. With $\varphi$ as a parameter, LD is installed in order to make the width of the projected beam to $25 \mathrm{~m}$, and the tipped angle of APD is 4 degrees. We measured the received SNR between $1 \mathrm{~m}$ point and 25 meter point at $1 \mathrm{~m}$ intervals using a miniature train. It can be seen from this result that even though the received SNR decreases gradually as the distance becomes longer, sufficient received SNR level can be obtained at $25 \mathrm{~m}$ point irrespective of $\varphi$. 
Furthermore, we can find that optimal value of $\varphi$ is 20 degrees. Therefore, we can say that a sufficient SNR can be obtained for the $25 \mathrm{~m}$ train length.

\subsection{Received signal power in a moving condition}

Measurement environment and result of the received signal power at moving situation are shown in Figure 7 with $\varphi=20$ and the tipped angle of APD of 4 . We measured the received signal amplitude in a moving condition between 1 meter point and $25 \mathrm{~m}$ point using a miniature train. It can be seen from this result that the received signal amplitude declines gradually when the train is moving. However, it is confirmed that the signal amplitude can be received continuously for the communication area.

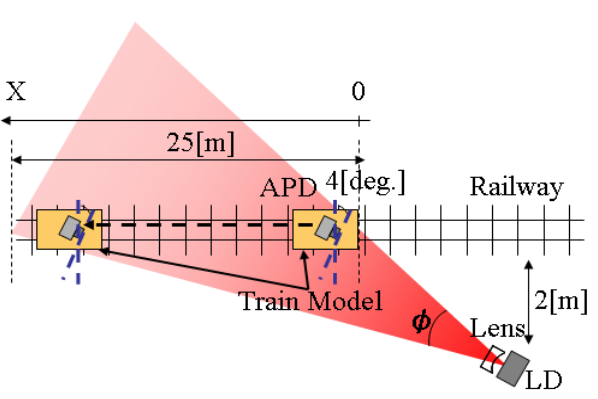

(Measurement)

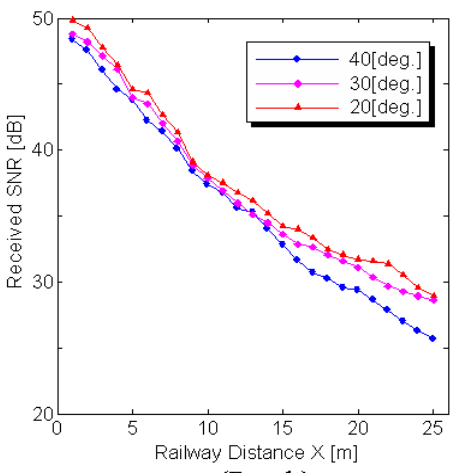

(Result)

Figure 6: Measurement and result of the received SNR in a stationary condition.

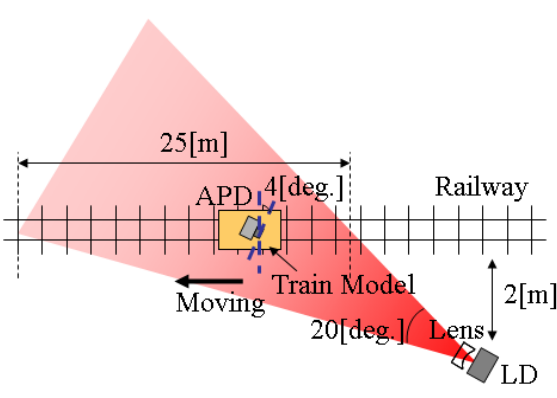

(Measurement)

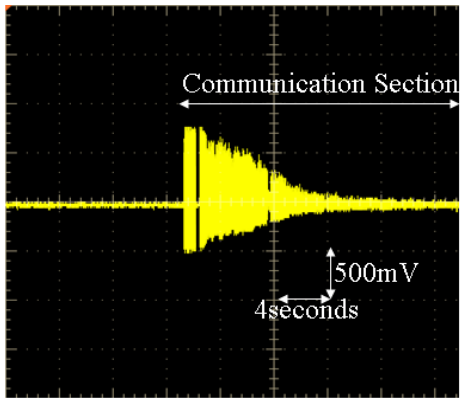

(Result)

Figure 7: Measurement and result of the received signal amplitude in a moving condition. 


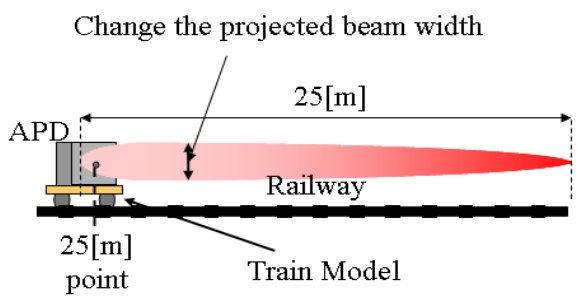

(Measurement)

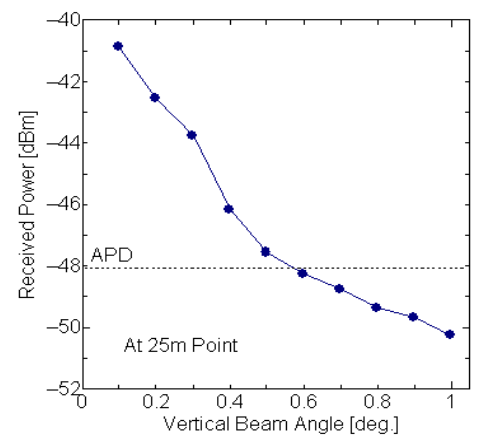

(Result)

Figure 8: Measurement and result of the received power at $25 \mathrm{~m}$ point with the expansion of vertical beam angle.

\subsection{Received power with the expansion of vertical beam angle}

When a train vibrates and shifts vertically, the sensor of APD sometimes jolt out of alignment from the projected beam. We measured received power at $25 \mathrm{~m}$ point with expanding the vertical beam angle of LD. Measurement environment and result of the received power at $25 \mathrm{~m}$ point with the expansion of vertical beam angle are shown in Figure 8 with $\varphi=20$ and the tipped angle of APD of 4 . A dotted line in Figure 8 shows the minimum sensitivity of APD assuming $1 \mathrm{GHz}$ optical signal. It can be seen from this result that at the vertical beam angle of 0.5 degrees the sufficient optical signal can be received. Therefore, we can say that by adjusting the vertical beam angle between 0.1 and 0.5 degrees, the vertical vibration and shift of a train can be absorbed.

\subsection{Outdoor experiment using test train}

We conducted the outdoor experiment using test train. We measured the received signal amplitude in the down-link and up-link when the train is moving at a speed of $15-20 \mathrm{~km} / \mathrm{h}$. Measurement environments of the outdoor experiment using test train are shown in Figure 9. Photograph of the outdoor experiment is shown in Figure 10. Table 3 shows experimental setup. In this experiment, a lens which works as optical concentrator and an interference filter are attached in front of APD. The length of platform is $20 \mathrm{~m}$ and that of test train is $19.6 \mathrm{~m}$. The width of the projected beam is $25 \mathrm{~m}$, the horizontal distance between LD and APD is 2 m. $\varphi=20$ degrees, and the tipped angles of APD is 14 degrees because Full Width at Half Maximum (FWHM) of lens is 17.3 degrees. Furthermore, the vertical beam angle is 0.3 degrees. Figure 11 shows the result of the outdoor experiment. It can be seen from these results that the received signal amplitude declines suddenly at the certain time when the train is moving. We think that these results from effect of FWHM of lens on sensor of APD and interference 
filter. However, it can be seen that the signal amplitude can be received continuously for $25 \mathrm{~m}$. Therefore, from these results, we can say that the proposed system will be a promising candidate for train communication.

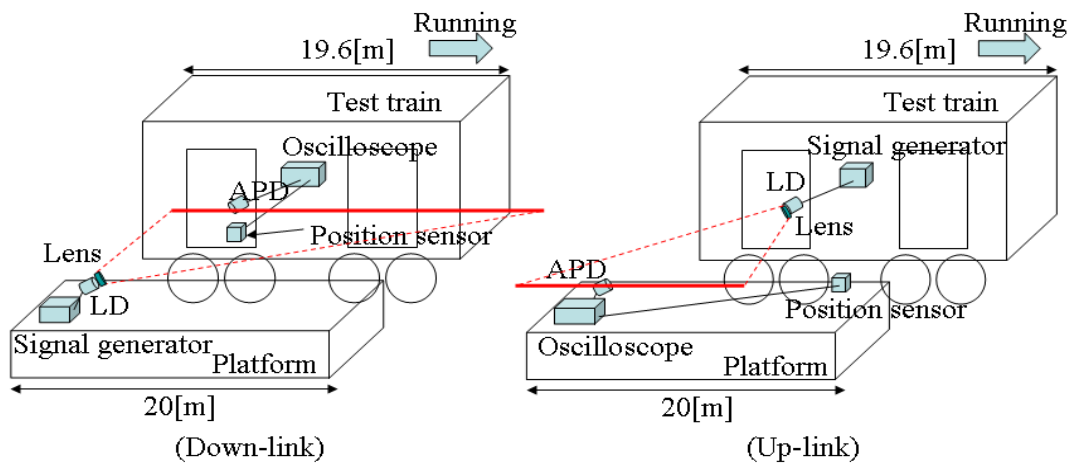

Figure 9: Measurements of the outdoor experiment using test train.

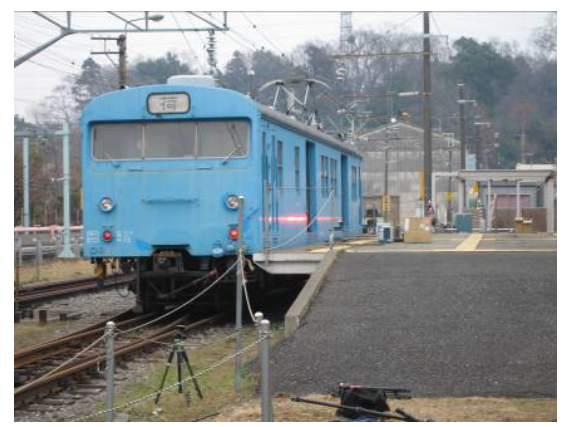

Figure 10: Photograph of the outdoor experiment.

Table 3: $\quad$ Experimental parameter of the outdoor experiment.

\begin{tabular}{|c|c|}
\hline Bit Rate & $100(\mathrm{Mbps})$ \\
\hline Transmitted power of LD & $100(\mathrm{~mW})$ \\
\hline Wavelength of LD & $670(\mathrm{~nm})$ \\
\hline Frequency of LD & $400(\mathrm{MHz})$ \\
\hline Horizontally spread beam angle (with lens) & $20($ degrees) \\
\hline Vertical beam angle & $0.3($ degrees$)$ \\
\hline Receiver & APD with lens \\
\hline Angle of receiver & $14($ degrees $)$ \\
\hline Diameter of receiver & $0.5(\mathrm{~mm})$ \\
\hline Material of cylindrical concave lens & Synthetic fused silica \\
\hline Weather & Fine day \\
\hline Received signal amplitude of ambient noise & $20(\mathrm{mV})$ \\
\hline
\end{tabular}



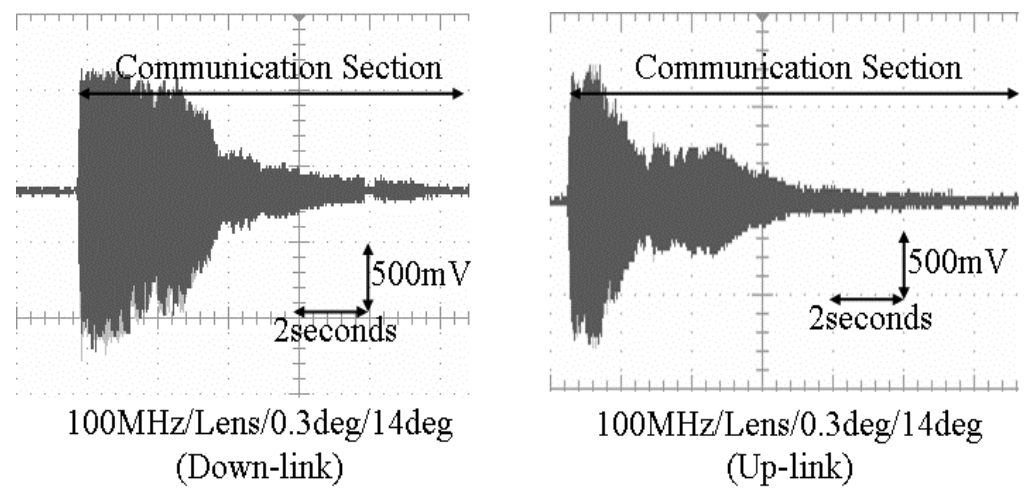

Figure 11: Results of the outdoor experiment.

\section{Conclusions}

In this paper, we proposed a new ground-to-train communication system using free-space optics technology in order to increase the transmission rate between a train and the ground. We investigated the system performance experimentally. It was shown from the experimental results that a sufficient SNR at stasis could be obtained for $25 \mathrm{~m}$ train length, and it was found that the optimal horizontal beam angle is 20 degrees. Moreover, the signal amplitude was more sufficient than the signal amplitude of ambient noise and a train can keep a communication link continuously for a $25 \mathrm{~m}$ train length. Furthermore, it was shown that by adjusting the vertical beam angle between 0.1 and 0.5 degrees, the effect of the vertical vibration and a shift of a train can be absorbed. Finally, we conducted the outdoor experiment using a test train. We believe that the proposed system is a promising candidate for train communication.

\section{References}

[1] Jun, H.W., \& Mei, K.K., Theory and analysis of leaky coaxial cables with periodic slots. Antennas and Propagation, IEEE Transactions on, Vol. 49, No.12, pp. 1723-1732, 2001.

[2] Nikura, H.., Akiyama, Y., \& Adachi, D., Train radio system with millimeter wave used for the Yamanashi maglev test line. RTRI Report, Vol. 15, No. $11,2001$.

[3] Komine, T. \& Nakagawa, M., Integrated system of white LED visible-light communication and power-line communication. Consumer Electronics, IEEE Transactions on, Vol. 49, No.1, pp. 71-79, 2003.

[4] Tanaka, Y., Komine, T., Haruyama, S. \& Nakagawa, M., Indoor visible light communication utilizing plural white LEDs as lighting. Personal, Indoor and Mobile Radio Communications, Vol. 2, pp. F-81-F-85, 2001. 
692 Computers in Railways X

[5] Kahn, J.M. \& Barry, J.R., Wireless infrared communications. Proc, IEEE, Vol. 85, No. 2, pp. 265-298, 1997.

[6] Barry, J.R., Wireless infrared communications, Kluwer Academic Publishers, Boston, MA, 1994. 